\title{
Computationally efficient modeling for assessing the energy efficiency of electric drivetrains using convex formulations
}

\author{
Matthias Vandeputte | Arne De Keyser | Guillaume Crevecoeur
}

Department of Electrical Energy, Metals, Mechanical Constructions \& Systems, Ghent University, B-9052 Zwijnaarde, Belgium

\section{Correspondence}

Matthias Vandeputte, Department of Electrical Energy, Metals, Mechanical Constructions \& Systems, Ghent University, B-9052 Zwijnaarde, Belgium. Email: matthias.vandeputte@ugent.be

\section{Funding information}

Strategic basic research project Emodo of the Flanders Make Strategic Research Centre for the Manufacturing Industry; BOF, Grant/Award Number: 01N02716, 01D37816; FWO research project, Grant/Award Number: G.0D93.16N

\begin{abstract}
High-fidelity models capturing the dynamical behavior can be engaged for the analysis of complex mechatronic systems. Determining the optimal control parameters and design characteristics of such systems necessitates solving multiple interconnected models acting on their respective physical domains and time scales. In this paper, high-fidelity physics-based models are constructed for several electrical subsystems. Loss mechanisms in the various components are inferred because these are key when performing optimal design and control in terms of energy-efficient conversion from power source to actuation. The complexity of the analyzed models is then reduced by introducing convex approximations for the occurring dissipation during power transfers, allowing abstracting the complicated dynamic behavior into a tractable convex formulation, specifically suited for time-efficient numerical simulation. The effectiveness of the strategy is demonstrated on a case study originating from the field of all-electric vehicles, embodying a series interconnection of a battery stack, a buck-boost converter, a voltage source inverter, and an asynchronous electric motor. Results show that the dynamic simulation of the proposed system, composed of multiple time scales, can be reliably computed using the composed convex mappings, hereby reducing the computational time approximately by a factor 461 , compromising only $1.8 \%$ accuracy regarding energy consumption assessment. The introduced convex formulation can therefore constitute the foundation for optimal control and design of complex mechatronic drives.
\end{abstract}

\section{KEYWORDS}

convex functions, loss mechanisms, mechatronics, numerical abstraction, optimal design

\section{1 | INTRODUCTION}

In recent years, the undeniable evidence for global warming became broadly accepted. Confining the detrimental effect on climate change for future generations subsequently turned into a central topic on the global scientific agenda. An important player regarding atmospheric pollution is the car and public transportation industry, that combined are responsible for approximately $23 \%$ of the world $\mathrm{CO}_{2}$-emissions. The vast increase of transportation emissions has always been driven by road transportation, indicated by a $68 \%$ increase in emissions since $1990 .^{1}$ As we can expect based on the contemporary evolution, future legislation will become even more demanding towards the manufacturers, while classical internal combustion-based propulsion is reaching its physical boundary in terms of energy efficiency. One can anticipate a trend towards better integration of electrical drivelines. 


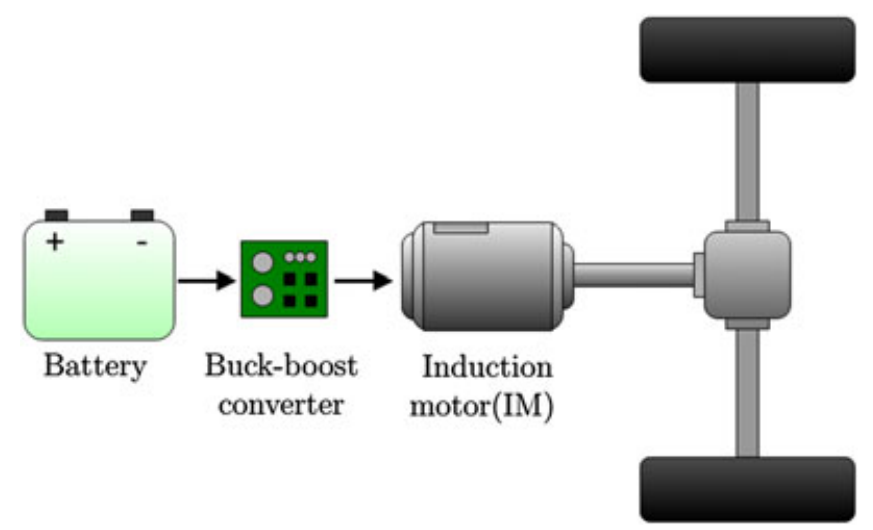

FIGURE 1 Overview of the electric drivetrain for vehicle propulsion. The induction motor is supplied by a 3-phase voltage source inverter

Electric drives for vehicle propulsion, and more generally electric power systems, introduce specific particularities, originating from the interaction between the different components on various time scales and physical domains. In this paper, a specific case study is introduced, demonstrated by the driveline in Figure 1, to propose a commonly applicable modeling strategy in electromechanical engineering. The proposed topology consists of a series connection of a battery stack ${ }^{2}$ to accommodate for the energy buffer, a buck-boost converter ${ }^{3}$ providing a controllable output direct current (DC)-level and a voltage source inverter ${ }^{4}$ that converts the DC-voltage to an appropriate 3-phase voltage system at the input of the induction motor. ${ }^{5}$

High-fidelity models for the various subsystems can be composed, including the dynamic behavior of the isolated components at the fastest time scale, hereby providing an accurate representation of the evolution during operation but necessitating small simulation time steps. Subsystems incorporating power electronic switches can be modeled by hybrid automata ${ }^{6}$ to fuse discrete events, ie, switching instants, and continuous dynamics, ie, internal state evolution, in a single modeling formalism. Furthermore, not all parts in electric drives can be represented by linear state space models and impose the need of local linearization procedures ${ }^{7}$ for each time instant, increasing the computational load. These characteristics render the dynamic simulation of electrical systems into a challenging and computationally expensive problem.

To reduce the appearing complexity of the problem, several approaches coexist. ${ }^{8}$ An appealing procedure introduces the power variables as the dynamic states of the system, abstracting the details and control protocols on the component level into their resulting power transfers. Power losses, assessed by high-fidelity simulations, are casted into an appropriate mapping. Within this approach, convex formulations prove to be useful. ${ }^{9}$ Convexity is however not assumed in advance but emerges as a consequence of the physical modeling procedure. The dissipation is casted into a convex regression model, and the general formulation of a convex problem statement 1 is attained with the matrices $\boldsymbol{A}_{\boldsymbol{e q}}$ and $\boldsymbol{B}_{\boldsymbol{e q}}$ representing the governing equality constraints, $g_{\mathrm{j}}(\cdot)(j=1, \ldots, m)$ denoting $m$ convex boundaries, and $\boldsymbol{P}$ the vector of the power flow variables in the system. The introduced inequality constraints originate from a relaxation of the corresponding equality relations, allowing the problem to be tackled by numerical convex solvers. The solution of the considered problem statement will naturally coincide with the boundary of the convex regions, hereby fulfilling the original equality constraints.

$$
\left\{\begin{array}{l}
\mathbf{A}_{\mathbf{e q}} \boldsymbol{P}=\mathbf{B}_{\mathbf{e q}} \\
g_{\mathrm{j}}(\boldsymbol{P}) \leqslant 0 \text { for } \mathrm{j}=1, \ldots, m .
\end{array}\right.
$$

The energy efficiency of drivetrains remains a focal point in both control and design applications. The introduced convex formulation can be directly engaged for optimal energy management, ${ }^{9}$ because of its time efficiency and reliability. Furthermore, it can constitute the basis for effective design methodologies, rendering the design of complex electronic systems tractable.

\section{2 | COMPONENT LEVEL SIMULATION OF THE ELECTRIC DRIVETRAIN}

Modeling of the different components is performed in state space representation, as to include most physical and dynamical particularities. Operation of the distinct components by their dedicated controllers is sketched. We consider the 
TABLE 1 Overview of the physical parameters in the drivetrain

\begin{tabular}{|c|c|}
\hline Quantity & Value \\
\hline \multicolumn{2}{|l|}{ Battery } \\
\hline$e_{0}$ & $1.2848 \mathrm{~V}$ \\
\hline$Q$ & $6.5 \mathrm{Ah}$ \\
\hline$R_{\mathrm{i}}$ & $0.0002 \Omega$ \\
\hline$K_{1}$ & $0.01875 \mathrm{~V}$ \\
\hline$K_{2}$ & $0.144 \mathrm{~V}$ \\
\hline$K_{3}$ & $2.3077(A h)^{-1}$ \\
\hline$n$ & 300 \\
\hline \multicolumn{2}{|l|}{ Buck-boost } \\
\hline$L$ & $130 \mu H$ \\
\hline$C$ & $5 \mathrm{mF}$ \\
\hline$R_{\mathrm{L}}$ & $0.0096 \Omega$ \\
\hline$R_{\mathrm{C}}$ & $0.005 \Omega$ \\
\hline \multicolumn{2}{|c|}{ Voltage source inverter } \\
\hline$R_{C E}$ & $0.005 \Omega$ \\
\hline \multicolumn{2}{|c|}{ Induction motor } \\
\hline$N_{\mathrm{p}}$ & 2 \\
\hline$R_{\mathrm{S}}$ & $0.03552 \Omega$ \\
\hline$R_{\mathrm{r}}$ & $0.02092 \Omega$ \\
\hline$L_{\mathrm{s}}$ & $15.435 \mathrm{mH}$ \\
\hline$L_{\mathrm{r}}$ & $15.435 \mathrm{mH}$ \\
\hline$L_{\mathrm{m}}$ & $15.100 \mathrm{mH}$ \\
\hline$J_{\mathrm{m}}$ & $0.75 \mathrm{kgm}^{2}$ \\
\hline \multicolumn{2}{|l|}{ Vehicle } \\
\hline$m$ & $1485 \mathrm{~kg}$ \\
\hline$J_{\mathrm{d}}$ & $1.2 \mathrm{kgm}^{2}$ \\
\hline$J_{\mathrm{w}}$ & $9.44 \mathrm{kgm}^{2}$ \\
\hline$i_{\mathrm{e}}$ & $1.47: 1$ \\
\hline
\end{tabular}

Numerical values for the battery and induction motor are based on Tremblay et $\mathrm{al}^{11}$ and Tabbache et al. ${ }^{21}$

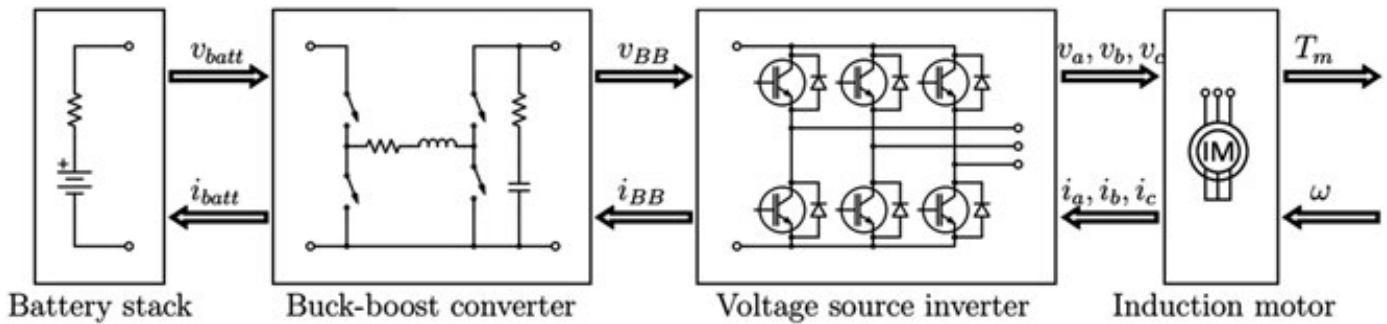

FIGURE 2 Information transfer between interconnected subcomponents for simulation of the drivetrain is based on their respective input and output ports

controllers as being distributed throughout the drivetrain, whereas their structure is fixed and dedicated for a certain component. As the work is focused on assessing the energy efficiency of the electric drivetrain, and thus the dissipation, the appropriate loss mechanisms in each subassembly are furthermore specified. The numerical values of the characteristic parameters of all components are provided in Table 1. A reviewed representation is depicted in Figure 2, focusing on the transfer of physical quantities between the distinct subsystems and denoting all incorporated notations.

\section{1 | Battery}

As a first approximation, a battery can be regarded in its ideal form as an ideal voltage source in series with a resistance in an electrical circuit. One of the major downsides of this method is that the dependency of the output voltage on the actual 
state of charge of the energy buffer is not modeled. The voltage-state-of-charge relation shows to be highly nonlinear for charging over around $80 \%$ of the maximum capacity or discharging under approximately $20 \%$ of the highest possible charge to be held by the battery. In Shepherd, ${ }^{10}$ these nonlinear sections are expressed as exponential curves added to the approximately linear voltage-discharge relation. For a known evolution of the current through the battery $\left(i_{\text {batt }}\right)$ in time $(t)$, this leads to

$$
e(t)=e_{0}-K_{1} \cdot \frac{Q}{Q-\int_{0}^{t} i_{\text {batt }}(\tau) \mathrm{d} \tau}+K_{2} \cdot \mathrm{e}^{-K_{3} \cdot \int_{0}^{t} i_{\text {batt }}(\tau) \mathrm{d} \tau}
$$

In this equation, $e(t)$ denotes the internal voltage of the battery cell with maximal charge $Q$ and corresponding voltage level $e_{0}$. The parameters $K_{1}, K_{2}$, and $K_{3}$ in the proposed expression can be deduced from data provided by the manufacturers. Incorporating the internal resistance $R_{\mathrm{i}}$, which is often nonnegligible and introduces dissipation, leads to an output voltage reduced by the corresponding voltage drop.

\section{2 | DC-DC converter}

One cannot suppose the output voltage of the battery to be constant. Current variations will induce a variable voltage drop over the internal resistance, which alters the voltage at the input of our system. ${ }^{11}$ Combined with the effect of falling voltage as the battery depletes during operation, one should anticipate these changes and allow to compensate them. As electrical motors operate more efficient at a constant voltage, a DC-DC converter (see Figure 3A with switches $S_{i}, i=1, \ldots, 4$ and parasitic resistances $R_{C}$ and $R_{L}$, capacitance $C$, and inductance $L$ ) should be considered in the proposed circuit. To deal with a broad range of voltage levels to the electric motor, a converter suited for both step-down $\left(\left[S_{1}, \overline{S_{2}}, S_{3}, \overline{S_{4}}\right] \leftrightarrow\left[\overline{S_{1}}, S_{2}, S_{3}, \overline{S_{4}}\right]\right)$ and step-up $\left(\left[S_{1}, \overline{S_{2}}, S_{3}, \overline{S_{4}}\right] \leftrightarrow\left[S_{1}, \overline{S_{2}}, \overline{S_{3}}, S_{4}\right]\right)$ conversions, buck and boost mode, respectively, is preferred.

A state space representation for a converter fed by a constant but variable voltage source at the input and connected to a variable current source at the output is derived, as illustrated in Figure 3. An approach that enables feasible assessment of the dynamic behavior constitutes of constructing an averaged dynamic model, spanning exactly one period. This concept can be implemented by approximating the state derivatives by the weighted sum of the state space representations belonging to the switching states, hence called the mean value model. The Boolean input to the system denoting the actual switching state is now replaced by the duty ratio $\delta$. Figure 3B illustrates the accuracy of the mean value model for the internal states (inductor current $i_{\mathrm{L}}$ and capacitor voltage $v_{\mathrm{C}}$ ) compared with the model simulating the full dynamics with inputs being the Boolean inputs representing the state of the distinct power electronic elements.

The proposed methodology results in an analytical representation of the converter in boost-operation:

$$
\begin{aligned}
& \boldsymbol{x}=\left[\begin{array}{c}
i_{\mathrm{L}} \\
v_{\mathrm{C}}
\end{array}\right] ; \boldsymbol{u}=\left[\begin{array}{c}
v_{\text {batt }} \\
i_{\mathrm{BB}}
\end{array}\right] ; \boldsymbol{y}=\left[\begin{array}{c}
i_{\text {batt }} \\
v_{\mathrm{BB}}
\end{array}\right] ; \\
& \mathbf{A}=\left[\begin{array}{cc}
-\frac{R_{\mathrm{L}}}{L}+\delta \frac{R_{\mathrm{C}}}{L} & -\delta \frac{1}{L} \\
\delta \frac{1}{C} & 0
\end{array}\right] ; \mathbf{B}=\left[\begin{array}{cc}
\frac{1}{L} & \delta \frac{R_{\mathrm{C}}}{L} \\
0 & -\frac{1}{C}
\end{array}\right] ; \mathbf{C}=\left[\begin{array}{cc}
1 & 0 \\
\delta R_{\mathrm{C}} & 1
\end{array}\right] ; \mathbf{D}=\left[\begin{array}{cc}
0 & 0 \\
0 & -R_{\mathrm{C}}
\end{array}\right] \text {. }
\end{aligned}
$$

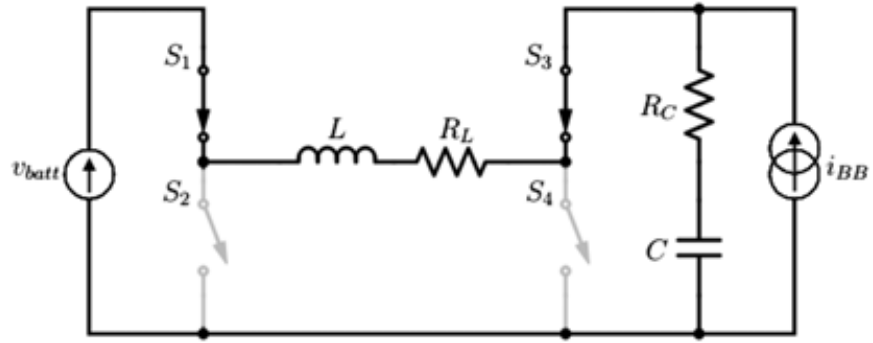

(A)DC-DC converter with voltage $\left(v_{\text {batt }}\right)$ and current source $\left(i_{\mathrm{BB}}\right)$.

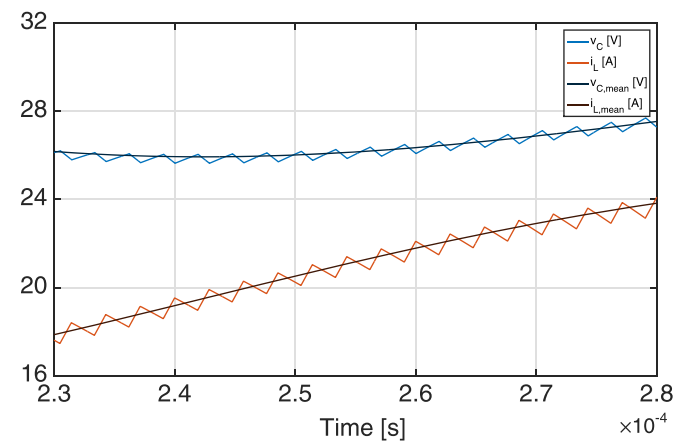

(B) Approximation by the mean value model

FIGURE 3 Physics-based implementation of the buck-boost converter 


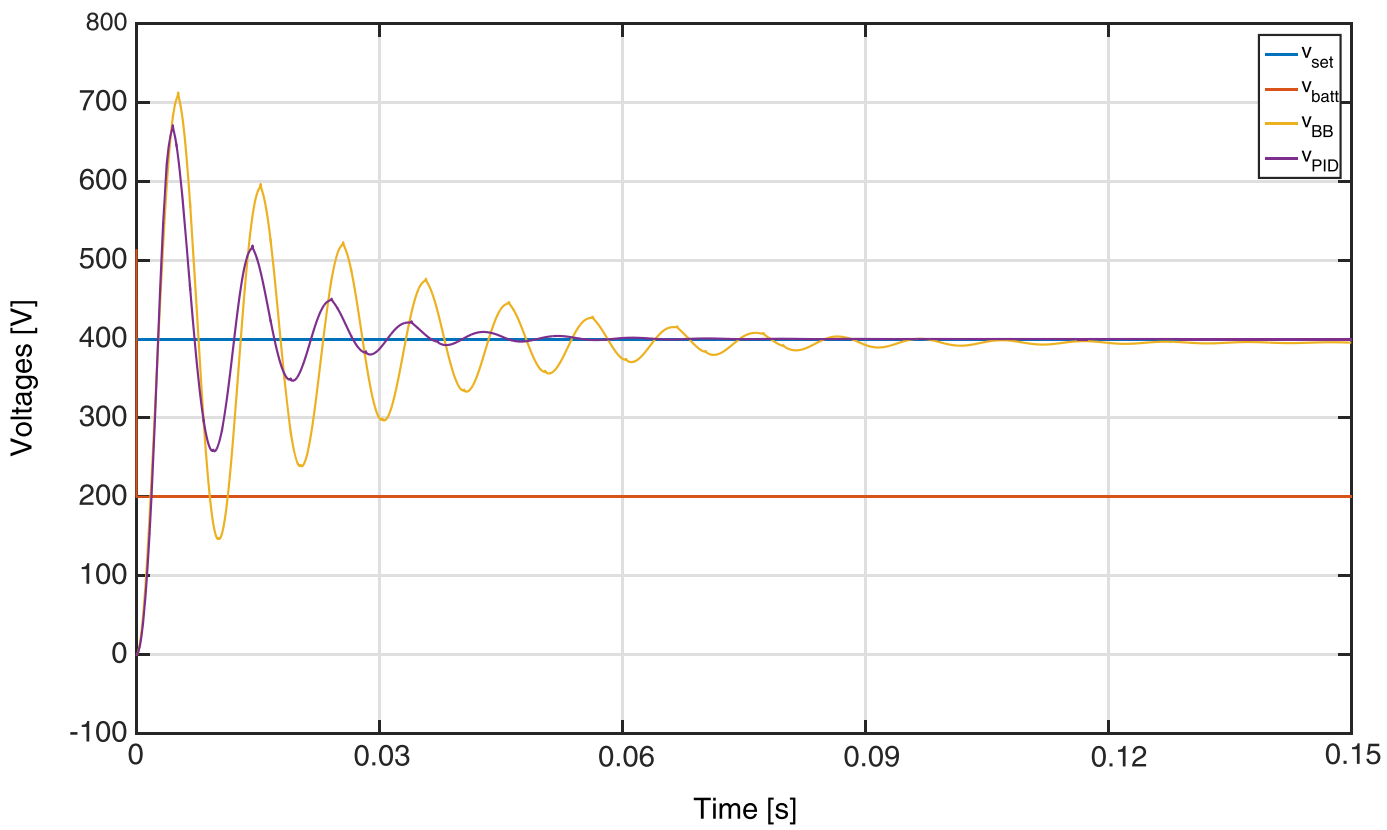

FIGURE 4 Comparison of the dynamic responses of the converter's voltage output in boost mode with and without proportional-integral-derivative (PID) control, with $v_{\text {set }}$ the voltage set point, $v_{\text {batt }}$ the voltage of the battery stack, $v_{\mathrm{BB}}$ the voltage at the direct current-bus, and $v_{\text {PID }}$ denoting the improved characteristic due to PID-control

With these matrices, the dynamic behavior of the converter is represented by the governing set of state space equations:

$$
\begin{aligned}
& \dot{x}=\mathrm{A} x+\mathrm{Bu} \\
& y=\mathrm{C} x+\mathrm{D} u .
\end{aligned}
$$

Equations for buck-mode can be deduced in a similar way. To control the transient behavior of the converter in a favorable manner and eliminate the steady-state error on the output voltage, a basic proportional integral derivative-control loop is implemented on the voltage $v_{\mathrm{BB}}$ with respect to an operational set point $v_{\text {set }}$. The corresponding gain factors can be determined by application of the Ziegler-Nichols design methodology. ${ }^{12}$ Remarkable improvements in the dynamical evolution of the DC-bus voltage can be observed in Figure 4. Both overshoot and settling time are reduced, while the steady-state error is eliminated. In the remainder of this work, a set point of $400 \mathrm{~V}\left(v_{\text {set }}=400 \mathrm{~V}\right)$ is assumed and the transient corresponding to a sudden step in the voltage reference is neglected. The incorporated control justifies the assumption of a constant voltage level at the DC-bus, as no step changes should be anticipated and the transient behavior is improved. Neglecting deviations from the ideal set point will nevertheless result in minor discrepancies at start-up of the electrical drive.

Note that the power electronic components introduce additional losses in the system, as the swift transition between states cannot be considered as a standard loss, called switching and conduction losses. ${ }^{13,14}$ Conduction losses are introduced by dissipation in an equivalent collector-emitter resistance $R_{C E}$, determined by the power electronic elements. Switching loss phenomena are captured by means of empirical models, given in Nicolai and Wintrich. ${ }^{15}$

\section{3 | Voltage source inverter}

The previously discussed DC-DC converter offers the system the possibility to operate at the most appropriate voltage level based on the actual operating conditions. In our work, we consider electric propulsion generated by a 3-phase alternating current (AC) motor, and therefore, the supplied DC voltage should be transformed to an appropriate rotating voltage system suited for the motor. Distinct possibilities coexist to satisfy this requirement, but the 3-phase voltage source inverter is elected with the torque control of the induction machine in perspective. The corresponding topology is represented in Figure 5. The output is constructed by passing the voltage to the proper phases using the provided switches. 


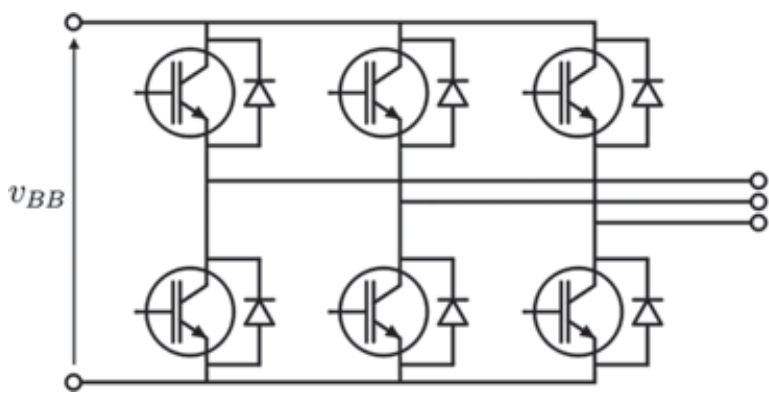

(A) Schematic of a basic 3-phase voltage source inverter

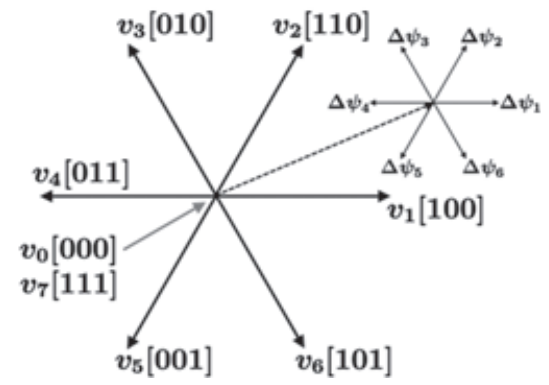

(B) Possible input vectors to the induction
machine

FIGURE 5 An external controller supplies the gates of the insulated-gate bipolar transistors with the proper switching signals. Freewheel diodes allow for reverse currents. Depending on the switching state, distinct phases are supplied. When all switches are in the same state, no voltage difference is observed in the motor frame

As the rapid transients can be considered hard to capture, the inverter will be modeled as an ideal conversion of a DC-voltage level into a 3-phase supply. Nevertheless, the losses in the power electronic components are evaluated and translated towards an equivalent voltage drop at the outlet.

\section{4 | Induction motor}

The driving component of our electrical system is chosen to be a standard AC induction machine. For the ease of simulation, a state space representation of the dynamics is derived. The dynamics of the induction machine are represented in a general way as follows:

$$
\boldsymbol{v}^{\mathbf{i}}=\mathbf{R}^{\mathbf{i}} \boldsymbol{i}^{\mathbf{i}}+\frac{d}{d t}\left[\mathbf{L}^{\mathbf{i}}(\theta) \boldsymbol{i}^{\mathbf{i}}\right]
$$

With $\boldsymbol{v}^{\mathbf{i}}$ and $\boldsymbol{i}^{\mathbf{i}}, i=1, \ldots, 6$ representing the stator and rotor phase systems, being the voltage vector and current vector for all 3 phases, respectively, $\mathbf{R}^{\mathbf{i}}$ the resistance matrix, and $\mathbf{L}^{\mathbf{i}}(\theta)$ the angle $(\theta)$ dependent inductance matrix of the asynchronous motor. The off-diagonal elements of the matrix $\mathbf{L}^{\mathbf{i}}(\theta)$ represent the respective mutual inductances between stator and rotor phases, while the diagonal entries denote the stator and rotor inductances. Starting from the provided set of differential equations, the dynamics of an induction machine can be converted into state space format by using the appropriate transformations and performing proper mathematical manipulations. ${ }^{16}$

$$
\mathbf{M} \dot{\boldsymbol{x}}=\mathbf{A}(\dot{\theta}) \boldsymbol{x}+\mathbf{B} \boldsymbol{u} .
$$

In problems dealing with stator control (eg, direct torque control), one usually adopts a stationary reference frame. Stator currents and fluxes make up the set of state variables $\boldsymbol{x}$, while the input vector $\boldsymbol{u}$ constitutes of the voltages in the proposed reference frame. The distinct matrices $\mathbf{M}, \mathbf{A}$, and $\mathbf{B}$ denote the state matrices of the global system, characterized by the physical stator and rotor resistances $R_{S}$ and $R_{r}$, and inductances $L_{s}, L_{r}$, and $L_{m}$, representing respectively the stator, rotor, and mutual inductance. The number of pole pairs is denoted by $N_{p}$, while $J_{m}$ represents the motor inertia. All numerical values can be found in Table 1 . To deliver the required torque within a reasonable region of accuracy, the direct torque control algorithm is implemented. ${ }^{17}$ Based on the current state of the phase angle and the magnitude of the flux, the most desirable input vector $\boldsymbol{v}_{\mathbf{i}}$ is supplied to the electric motor. As the inverter contains 3 pairs of switches, 8 different combinations can be constructed, see Figure 5. The flux $\psi$ is ought to evolve in the direction of the voltage vector, and in this way, the output torque and flux magnitude can be regulated to be confined in a limited hysteresis region around the set point. The desired magnitude of the flux is here nevertheless fixed at $1.0 \mathrm{~Wb}$.

\section{5 | Vehicle dynamics}

To propel the vehicle, different dynamic mechanical resistances have to be overcome, resulting in a counteracting torque. One can categorize 2 main components in the required torque, being the rolling, drag, and inclination resistances imposed 
by the environment and the acceleration torque caused by the inertia of the vehicle. Based on the desired acceleration, the internal control system can deduce the torque required to accelerate at a given rate, while the road dynamics impose the different counteracting forces. The considered vehicle is characterized by an inertia for the wheels $\left(J_{w}\right)$, a drive inertia $\left(J_{d}\right)$, and a total mass $(m)$. The final reduction in the driveline is abbreviated as $i_{e}$ and is fixed. For further information, one can consult Gillespie. ${ }^{18}$

\section{3 | CONVEX MAPPING FORMULATION}

The previously elaborated state space formulations allow to simulate the drivetrain design with a high accuracy and reliability but at the cost of an extensive need of computational resources and time. A more efficient representation of the interconnected system is strongly desired and the included models need to be casted in an appropriate format. Nevertheless, transient behavior is ought to be incorporated in the proposed abstraction.

\section{1 | Convex loss models}

Convex reformulations of existing problems provide a helpful means in the pursuit of time-effective simulation of power flow-based systems as this enables efficient solution algorithms and thereby elevated computational gains. ${ }^{8}$ The focal point of most electrical drives consists of improved energy management of the accommodated energy buffers as to maximize the energy efficiency in operation. Intuitively, one could expect a convex behavior of the dissipated power when deviations from optimal operation occur, as losses need to increase in both directions. This mindset constitutes the foundation for the introduced abstractions in Egardt et al. ${ }^{9}$ In this paper, convexity is not assumed a priori but intervenes as a consequence of the physical behavior of the distinct components on the level of power transfers.

If one considers the dynamic evolution of the buck-boost converter 3 combined with the constitutional law of the battery stack and its internal resistance, the desired convexity of the dissipated power flows can be mathematically derived. For regime conditions, which corresponds to a fixed state trajectory and thus no deviations in state variables over time $(\dot{x}=0)$, the following set of equations is deduced:

$$
\left\{\begin{array}{l}
\delta i_{\text {batt }}=i_{\mathrm{BB}} \\
v_{\mathrm{C}}=-\frac{R_{\mathrm{L}}}{\delta} i_{\mathrm{L}}+R_{\mathrm{C}} i_{\mathrm{L}}+\frac{v_{\text {batt }}}{\delta}+R_{\mathrm{C}} i_{\mathrm{BB}} \\
i_{\text {batt }}=i_{\mathrm{L}} \\
v_{\mathrm{BB}}=\delta R_{\mathrm{C}} i_{\mathrm{L}}+v_{\mathrm{C}}-R_{\mathrm{C}} i_{\mathrm{BB}} \\
v_{\text {batt }}=E_{0}-R_{\mathrm{i}} i_{\text {batt }}
\end{array} .\right.
$$

The total internal voltage $E_{0}$ is uniquely defined by the number of battery cells $n$, and the internal voltage of a single battery $e_{0}$ as a series connection within the buffer pack is assumed.

$$
E_{0}=n e_{0} .
$$

After some mathematical manipulations, expressions for the power flows are obtained, which appear to be functions of $E_{0}, i_{\mathrm{BB}}$, and $i_{\text {batt }}$.

$$
\left\{\begin{array}{l}
P_{\text {in }}=E_{0} i_{\text {batt }} \\
P_{\text {out }}=i_{\mathrm{BB}} v_{\mathrm{BB}}=R_{\mathrm{C}} i_{\mathrm{BB}}^{2}+R_{\mathrm{C}} i_{\mathrm{BB}} i_{\text {batt }}-\left(R_{\mathrm{i}}+R_{\mathrm{L}}\right) i_{\mathrm{batt}}^{2}+E_{0} i_{\text {batt }}
\end{array} .\right.
$$

The different currents can be interpreted as being averaged values over a prolonged time interval. In the actual determination of the loss models, the power flow variables are nevertheless calculated by considering the instantaneous values for the underlying physical quantities and averaging the corresponding power losses. Note that averaging the instantaneous state variables results in a slight underestimation of the power losses in the components. This approximation will however constitute as a valid base for validating the convexity of the dissipated power as a function of the output power. The internal components, eg, the DC-bus capacitance $C$, are designed such that the relevant state variables have a relatively low variability in the span of one duty cycle. Considering the case of the DC-bus capacitance, its value is chosen such that the DC-bus voltage $v_{\mathrm{BB}}$ can be roughly considered constant. For the dissipated power follows 


$$
P_{\mathrm{d}}=P_{\text {in }}-P_{\text {out }}=-R_{\mathrm{C}} i_{\mathrm{BB}}^{2}-R_{\mathrm{C}} i_{\mathrm{BB}} i_{\text {batt }}+\left(R_{\mathrm{i}}+R_{\mathrm{L}}\right) i_{\text {batt }}^{2}
$$

Referring to the flows depicted in Figure 2, one can consider the quantity $i_{\mathrm{BB}}$ as an external factor, imposed by the remainder of the complete system. Furthermore, deviations in $E_{0}$ are generally negligible with respect to the other parameters, given that the time constant of depletion is much larger than the duration of the electrical transients. These parameters are therefore regarded as instantaneously constant quantities. A sufficient condition to ensure the convexity of the power losses $P_{\mathrm{d}}$, originating in both the battery stack and the converter, as a function of the output power $P_{\text {out }}$ is formulated as

$$
\frac{\mathrm{d}^{2} P_{d}}{\mathrm{~d} P_{\text {out }}^{2}} \geqslant 0
$$

In the considered case, this leads to

$$
\begin{aligned}
\frac{\mathrm{d} P_{\mathrm{d}}}{\mathrm{d} P_{\text {out }}} & =\frac{\mathrm{d} P_{\mathrm{d}}}{\mathrm{d} i_{\text {batt }}} \cdot \frac{\mathrm{d} i_{\text {batt }}}{\mathrm{d} P_{\text {out }}} \\
& =\frac{2\left(R_{\mathrm{i}}+R_{\mathrm{L}}\right) i_{\text {batt }}-R_{\mathrm{C}} i_{\mathrm{BB}}}{-2\left(R_{\mathrm{i}}+R_{\mathrm{L}}\right) i_{\text {batt }}+R_{\mathrm{C}} i_{\mathrm{BB}}+E_{0}} .
\end{aligned}
$$

For the second derivative with respect to $P_{\text {out }}$, one gets

$$
\frac{\mathrm{d}^{2} P_{\mathrm{d}}}{\mathrm{d} P_{\text {out }}^{2}}=\frac{2 E_{0}\left(R_{\mathrm{i}}+R_{\mathrm{L}}\right)}{\left(-2\left(R_{\mathrm{i}}+R_{\mathrm{L}}\right) i_{\text {batt }}+R_{\mathrm{C}} i_{\mathrm{BB}}+E_{0}\right)^{3}}
$$

and consequently, given that the resistances and internal voltage are all positive real numbers,

$$
i_{\text {batt }} \leqslant \frac{R_{\mathrm{C}} i_{\mathrm{BB}}+E_{0}}{2\left(R_{\mathrm{i}}+R_{\mathrm{L}}\right)} \approx \frac{E_{0}}{2\left(R_{\mathrm{i}}+R_{\mathrm{L}}\right)} .
$$

To prove the asserted convexity property, it suffices to note that the occurring parasitic resistances are commonly very small in realistic scenarios $\left(R_{\mathrm{i}}, R_{\mathrm{L}}, R_{\mathrm{C}} \sim \mathrm{m} \Omega\right.$ ), while the internal voltage adds up to values in the order of $300 \mathrm{~V}$, because all battery cells are connected in series. Taking these considerations into account, the validity of the condition 14 can be guaranteed in practical circumstances, as current is limited because of heating effects. Additionally, the battery contains an important energy buffer, which cannot be neglected in power flow evaluations and introduces an additional degree of freedom for the loss mapping.

Based on the previous discussion, a steady-state representation of the battery and DC-DC converter can be casted in a convex format. By including the dynamical behavior in this mapping procedure, these metamodels, ie, regression models that are based on physics-based models, are directly engaged in the analysis of the interconnected drivetrain. Power electronic losses, both conduction and switching losses, are assessed by introducing a resistance for the current flow and empirical formulations for the dissipated energy during switching events. The convexity of the system is not disturbed by the presence of switching elements, as the losses remain constrained. Fitting a parabolic regression model to the data obtained by numerical simulations of the high-fidelity loss models results in the convex surface shown in Figure 6A for a battery stack consisting of 300 cells.

Dissipation in the propelling mechanisms of the drivetrain, namely, the asynchronous motor and its 3-phase supply, can be treated equivalently. Drawing the analogy with bond graph representation, the mapping needs to be constructed for a constant flow input, corresponding to the rotational speed of the motor axle. As no significant mechanical means of energy storage are present in the considered case study, no additional dimension needs to be included. In the proposed case study, an induction motor with a rated power of $75 \mathrm{~kW}$ is casted into its representative loss formulation, which is demonstrated in Figure 6B. It is important to stress that the convex behavior is only observed in the $P_{\text {out,IM-direction, as }}$ is required to fulfill the conditions of the convex problem formulation. 


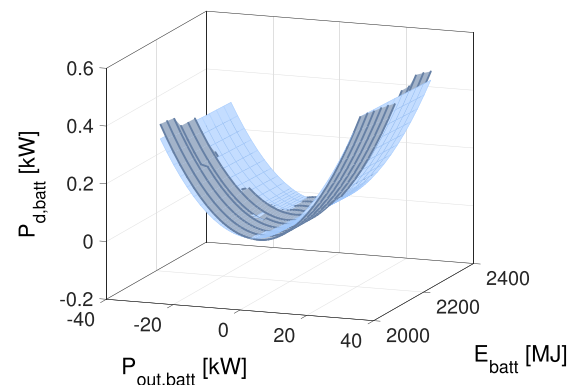

(A) Convex mapping for the dissipation in the battery stack and DC-DC converter.

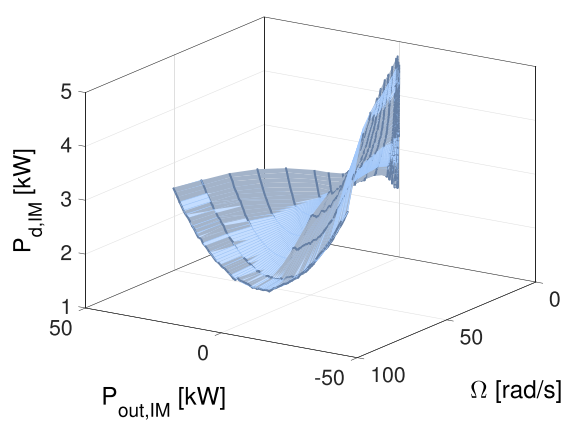

(B) Convex mapping for the dissipation in the VSI and induction motor for various mechanical speeds $\omega$.

FIGURE 6 Convex regression models for the power dissipation in the distinct subsystems within the drivetrain. DC, direct current; VSI, voltage source inverter

\subsection{Convexification of the vehicle dynamics}

We reconsider the vehicle dynamics, consisting of the rolling resistance, drag force, road inclination, and acceleration torque, and format them into an accessible shape within our convex framework. Taking a fixed road and speed profile into account, all required forces can be readily deduced. Therefore, the vehicle is split up in 2 modules: the rotating driveline and the vehicle body, as schematically represented in Figure 7. This way the driveline efficiency and the losses by forces acting on the outside of the vehicle are easily derived and split up in an intuitive fashion.

The rotational kinetic energy stored in the drivetrain is obtained as follows, based on the rotational speed $\omega$ of the shaft demonstrated in Figure 2:

$$
E_{\text {kin, drive }}=\frac{1}{2} J \omega^{2}
$$

With $J$ being the rotational inertia of the driveline components translated to an equivalent inertia at the wheel axle,

$$
J=J_{w}+i_{e}^{2} J_{d}+i_{e}^{2} J_{m}
$$

where $i_{e}$ is the gear ratio of the rear differential and $J_{m}, J_{d}$, and $J_{w}$ the inertia of the motor, the driveline, and the wheels, respectively.

A generally acceptable approach is to assume a fixed efficiency of the driveline, eg, $\eta=90 \%$. In the case that the wheels are driven by the motor, mechanical power is transferred to the wheels and part of the motoring power is lost in the driveline.

$$
P_{\mathrm{d}, \mathrm{drive}}=P_{\text {in,drive }}-P_{\text {out }, \mathrm{drive}}=(1-\eta) P_{\text {in, drive }}
$$

When motoring, the input and output powers are positive by sign convention. Equivalently, when kinetic mechanical energy is recovered and the induction motor (IM) is employed as a generator unit, both input and output power flows of the driveline are negative, resulting in the following expression for driveline losses:

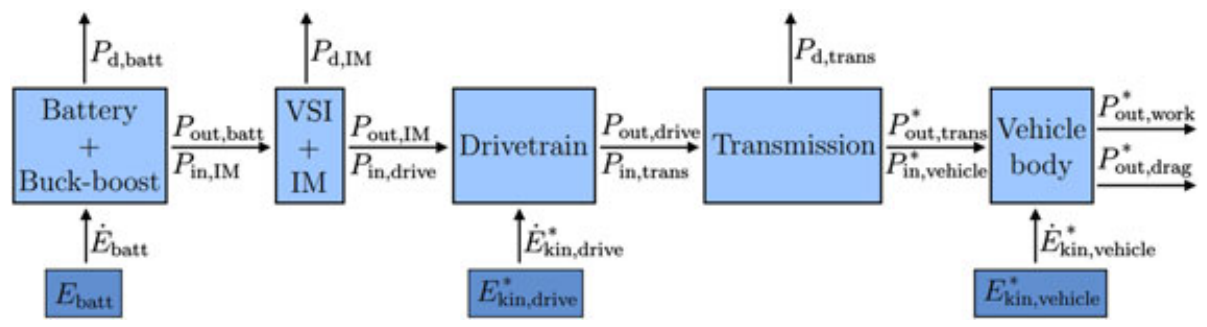

FIGURE 7 Schematic overview of the power flows in the drivetrain. Power variables marked by * are uniquely determined by the speed profile of the driving cycle. IM, induction motor; VSI, voltage source inverter 


$$
P_{\mathrm{d}, \text { drive }}=P_{\text {in,drive }}-P_{\text {out,drive }}=\left(1-\frac{1}{\eta}\right) P_{\text {in,drive }} .
$$

As a general expression for the transmission losses, we state

$$
P_{\text {d,drive }}=\max \left((1-\eta) P_{\text {in,drive }},\left(1-\frac{1}{\eta}\right) P_{\text {in,drive }}\right) .
$$

One is now free to consider the external loss functions, rolling resistance and drag force, as a fixed loss or output power flow. Note that there is no optimization needed in this module of the vehicle assembly. All power flows and buffer states result directly from the road profile. The input power flow for the driveline is a result of the 2 sets of equations following from the conservation of energy for power flows passing through both mechanical sections.

\section{3 | Convex problem formulation}

The power flows in the system are schematically visualized in Figure 7, hereby engaging the previously discussed convexification of the energy dissipation of the components (as described in Section 2) in the electric drivetrain. Interconnection between the different electrical subsystems is governed by equality constraints, representing a physical connection or the power balance for a single component.

The general power node equation is written as

$$
P_{\text {in }}+\dot{E}=P_{\text {out }}+P_{\text {d }} .
$$

Herein, the input, output, and dissipated powers are represented by $P_{\text {in }}, P_{\text {out }}$, and $P_{\mathrm{d}}$, respectively, while the notation $\dot{E}$ symbolizes the change in internally stored energy. To translate the considered problem into a suitable format matching 1 , this last class of equality constraints 20 is relaxed to obtain the appropriate convex inequality constraints. Figure 7 shows that this abstraction is equivalent for all components in which energy is dissipated. For the other system components, the proposed relaxation is not necessary as only 2 power flow state variables are to be determined and can be connected by a linear equality constraint, satisfying the convex optimization structure. In the case of the assembly containing the battery and the converter, this consequently leads to

$$
\dot{E} \geqslant P_{\text {out }}+P_{\text {d }},
$$

and for the electrical motor and its drive, in which the energy buffer does not depend on the dynamic power flow variables, one can write

$$
P_{\text {in }} \geqslant P_{\text {out }}+P_{\text {d }}
$$

Assume $\tilde{P}_{\text {in }}$ to be the optimal solution for $P_{\text {in }}$ coinciding with the convex inequality constraint and another solution $P_{\text {in }}$ satisfying the inequality constraint 22 :

$$
\begin{aligned}
P_{\text {in }} & =\gamma+P_{d}\left(P_{\text {in }}\right)+P_{\text {out }} \\
& =\gamma+P_{d}\left(\tilde{P}_{\text {in }}+\gamma\right)+P_{\text {out }} \\
& =\gamma+P_{d}\left(\tilde{P}_{\text {in }}\right)+\Delta P_{d}+P_{\text {out }} .
\end{aligned}
$$

With $\gamma$ a nonnegative real-valued slack variable and thus

$$
P_{\text {in }}=\tilde{P}_{\text {in }}+\gamma+\Delta P_{\text {d }}
$$

For which $\tilde{P}_{\text {in }}$ is optimal if $\gamma+\Delta P_{\mathrm{d}} \geqslant 0$ and consequently the relaxed convex problem conversion results in a solution coinciding with the inequality constraint and thus satisfying the original equality constraint.

It can easily be shown that

$$
\Delta P_{\mathrm{d}} \leqslant \gamma\left[\frac{\partial P_{\mathrm{d}}}{\partial P_{\text {in }}}\right]_{\max }
$$

A sufficient condition for the applicability of the aforementioned relaxation is that $-1 \leqslant \frac{\partial P_{\mathrm{d}}}{\partial P_{\mathrm{in}}} \leqslant 1$ for the whole operating range of the system. This condition is intuitively weak, as it would be of no use to increase the transferred mechanical power if it results in an added dissipation greater than the added mechanical power. 
Extrapolating the proposed relaxation theorem to all assemblies within the series connection, a convex formulation of the dynamical problem introduced by the state space modeling is obtained, satisfying the requirements of 1 . The occurring power variables constitute the state vector $\boldsymbol{P}$ at each time instant $t_{\mathrm{k}}(k=1, \ldots, N)$.

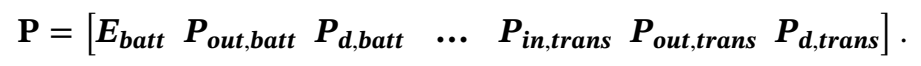

Based on the known time evolution of the demanded power at the wheels $\boldsymbol{P}_{\text {out,work }}$ and $\boldsymbol{P}_{\text {out,drag, }}$, which follows from solving the problem concerning vehicle dynamics, all power flows can be determined by solving the following set of equations, with $\Delta t$ representing the employed simulation time step:

$$
\left\{\begin{array}{l}
P_{\text {in,IM }}(k)-P_{\text {out,batt }}(k)=0 \\
P_{\text {in,drive }}(k)-P_{\text {out }, I M}(k)=0 \\
P_{\text {in,trans }}(k)-P_{\text {out,drive }}(k)=0 \\
P_{\text {in,vehicle }}(k)-P_{\text {out,trans }}(k)=0 \\
P_{\text {in,vehicle }}(k)=P_{\text {out,work }}(k)+P_{\text {out,drag }}(k)-\dot{E}_{\text {kin,vehicle }}(k) \\
P_{\text {in,drive }}(k)-P_{\text {out,drive }}(k)=-\dot{E}_{\text {kin,drive }}(k) \\
P_{\text {d,batt }}\left(E_{\text {batt }}(k), P_{\text {out,batt }}(k), k\right)+P_{\text {out,batt }}(k)-\frac{E_{\text {batt }}(k)-E_{\text {batt }}(k-1)}{\Delta t} \leqslant 0 \\
P_{\text {d,IM }}\left(P_{\text {out,IM }}(k), k\right)+P_{\text {out,IM }}(k)-P_{\text {in,IM }}(k) \leqslant 0 \\
P_{\text {d,trans }}\left(P_{\text {out,trans }}(k), k\right)+P_{\text {out,trans }}(k)-P_{\text {in,trans }}(k) \leqslant 0
\end{array} .\right.
$$

With the introduction of the appropriate matrices, 27 can be translated into a completely equivalent formulation as in 1. The elements of the matrix $\mathbf{P}$ are arranged in the left-hand side of the equality constraints in 1 , while the inequality constraints introduce the previously constructed convex loss relations.

\section{4 | RESULTS AND DISCUSSION}

The derived convex formulation of the electrical drivetrain copes with the dynamical evolution of the interconnected system by validating all power equations, governing the transfer between different assemblies within the total configuration. The validity of the proposed abstractions needs to be assessed in a dynamical context before it is used in a reliable manner as a valuable substitute in the dynamical power flow simulation of electromechanical drives. Its applications do not remain limited to simulation purposes solely, as the considered strategy can constitute the basis for time-effective optimal design problems, which are not tractable for standard contemporary solvers in a numerical environment.

The complete drivetrain of Figure 1 is simulated for a prolonged time horizon, based on the interconnection of the separate subsystems. All dynamic particularities are introduced by considering the physical state space representations. As the switching frequency of the buck-boost converter is arbitrarily chosen to be $20 \mathrm{kHz}$, the minimal time step is fixed at $50 \mu \mathrm{s}(\Delta t=50 \mu \mathrm{s})$. Torque and speed references are provided by the urban driving cycle, ${ }^{19}$ a standardized cycle within European legislation to evaluate the energy consumption of passenger cars in urban areas. The imposed trajectory is visualized in Figure 8.

As the drivetrain is implemented in a discrete computational environment, the continuous-time state space formulations of 4 and 6 are replaced by their respective approximations in first-order forward differencing 28 . The stability of this approach is further discussed in Benigni and Monti. ${ }^{20}$

$$
\boldsymbol{x}_{\mathbf{k}+\mathbf{1}}=\left(\mathbf{I}_{\mathbf{n}}+\Delta t \cdot \mathbf{A}\right) \boldsymbol{x}_{\mathbf{k}}+\Delta t \cdot \mathbf{B} \boldsymbol{u} .
$$

The same problem statement is solved by using the convex formulation obtained in 27. A much coarser simulation is now possible as all control protocols are abstracted in the metamodel and one does not have to account directly for the separate switching events. All dynamical details are included in the appropriate convex loss mappings, combining the distinct time scales from the original problem into a single formulation. The main trade-off consists of determining the optimal approximation, as a more extensive convex basis than in Figure 6 allows for close approximations but necessitates a higher evaluation time.

To validate the reliability of the proposed methodology, one can compare the response with respect to the energy losses in the distinct electrical systems. Practical applications of the power flow convexification can be found in both optimal control and design problems, which are possibly heavily interconnected. The main optimization objective will then deal 


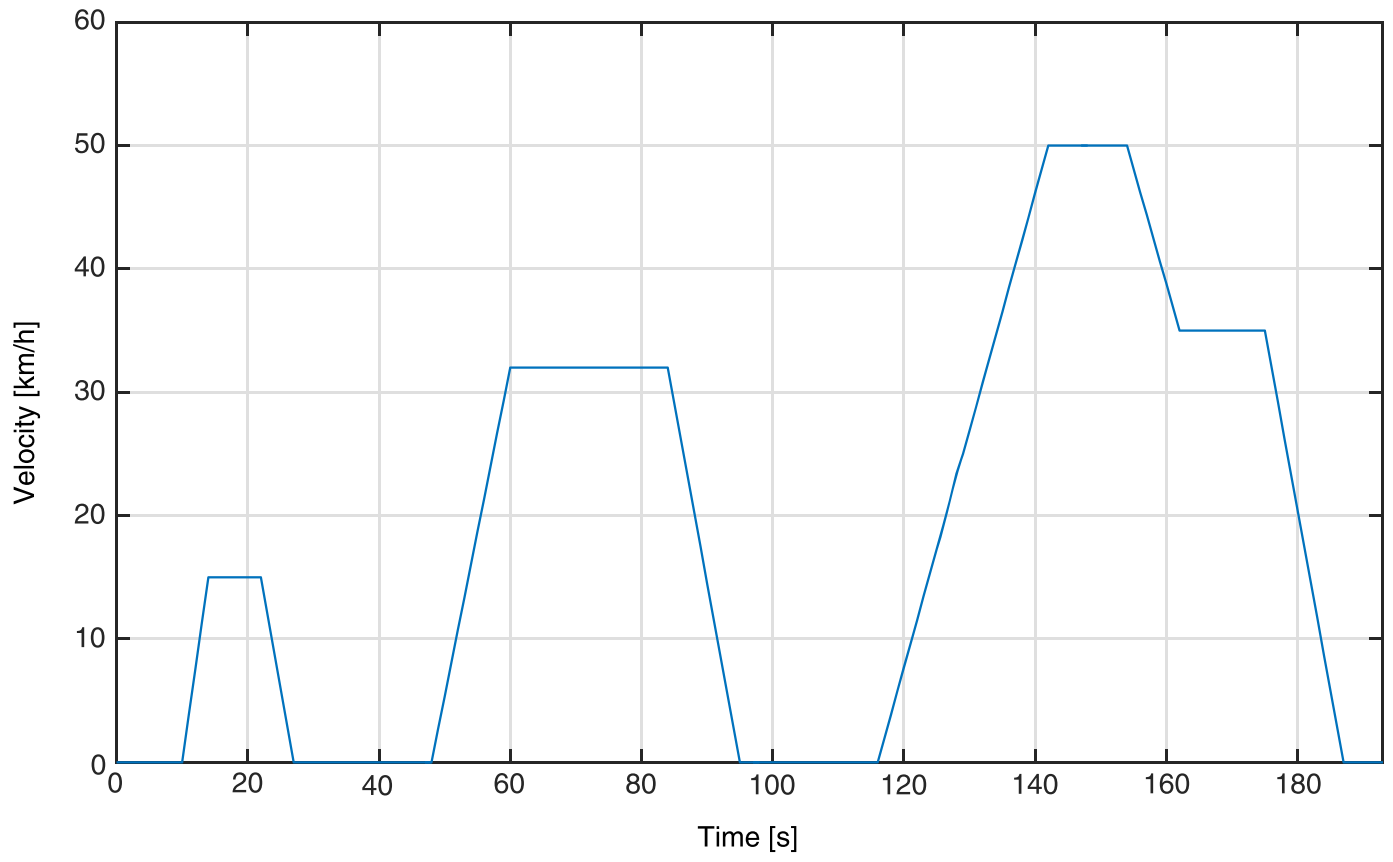

FIGURE 8 Velocity profile of the reference driving cycle (urban driving cycle)

with the energy consumption of the complete topology, as this will determine the operational range of the all-electric vehicle and define its economical viability. Therefore, special focus is devoted to the evolution of the battery charge over time. A comparison between the high-fidelity simulation, based on the physical representations, and the convex approximations for a time step of 1 second is provided in Figure 9A. Execution of the physical model requires 30 minutes 5 seconds of CPU-time, whereas 3.91 seconds was needed when using the convex approximations of the power flows, giving rise to an acceleration of 461 in computational efficiency. Computations were performed on a standard computer equipped with an Intel Core i7-6600U processor and a 64-bit operating system. Furthermore, a modeling accuracy of $98.2 \%$ is still maintained. Denoting the values obtained by the regression model at time instant $k$ with a hat, ie, $\hat{\mathrm{E}}_{\text {batt }}(k)$ for $k=1, \ldots, N$, the modeling accuracy is defined as

$$
\operatorname{Accuracy}(\%)=100\left(1-\frac{1}{N} \sum_{k=1}^{N} \frac{\left|E_{\text {batt }}(k)-\hat{\mathrm{E}}_{\text {batt }}(k)\right|}{\left|E_{\text {batt }}(k)-E_{\text {batt }}(0)\right|}\right) .
$$

To increase the truthfulness of the simulation, one can opt to decrease the simulation step size in the convex framework. The influence of this parameter is depicted in Figure 9B. As in the convex mappings, the impact of the lowest order time scale is abstracted by averaging the losses over a representative time horizon; the upper limit to the accuracy of the methodology is not imposed by the magnitude of the time step in the convex framework but rather by the accuracy of the empirical loss estimation. The quality of the energy assessment procedure is therefore only slightly dependent on the incorporated time step as can be observed by the nonmonotonic behavior of the curve in Figure 9B.

A strong resemblance between both curves of Figure 9A regarding the energy buffer within the configuration can be observed. More accurate models can be readily constructed by introducing convex terms with a higher complexity, but at the cost of increased computational needs. The respective loss approximations for the battery stack and asynchronous drive are highlighted in Figure 10. As the transient behavior is not considered in the convex approximations, swift dynamic transitions are not predicted into detail by the proposed methodology. The evolution of the power flow variables is therefore smoothened throughout time, and the power peaks observed in the high-fidelity simulation are discarded. The overall trend is nevertheless clearly incorporated in both time-efficient loss models.

For general design problems, the obtained accuracy suffices and the gain in calculation time to evaluate the effectiveness of a single design considerably outweighs the minor loss in accuracy. Furthermore, the number of variables in power flow optimization rises exponentially with both numbers of components and considered time interval, rendering the design problem intractable for classical algorithms. Casting the monitored problem into a convex alternative thus proves to be a useful tool in the design of more complex systems, embodying several electrical and mechanical subsystems interacting on their respective time scales. 


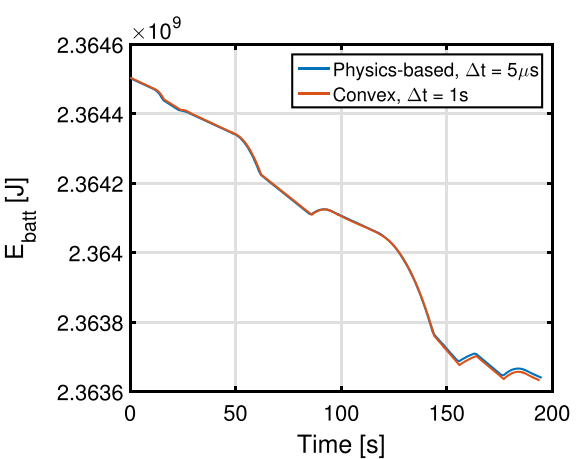

(A) Comparison of the battery charge evolution for both approaches.

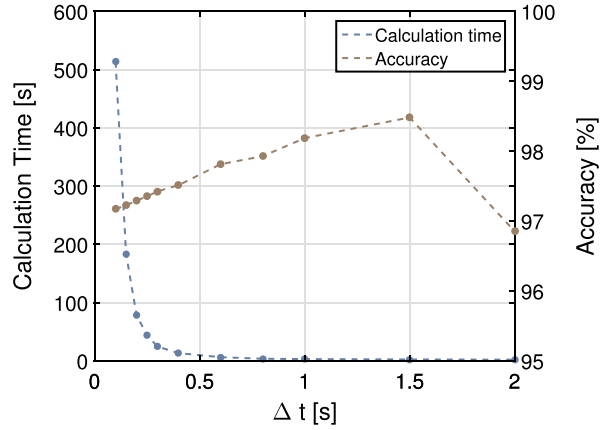

(B) Calculation time and accuracy of the convex abstraction for various time steps.

FIGURE 9 Accuracy of the convex approximations for the battery charge in the buffer

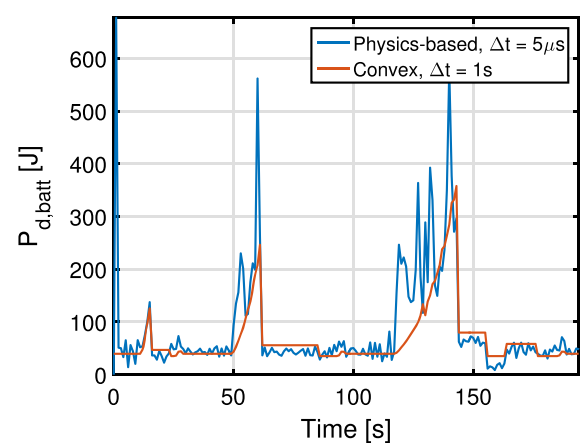

(A) Dissipated power in the battery stack.

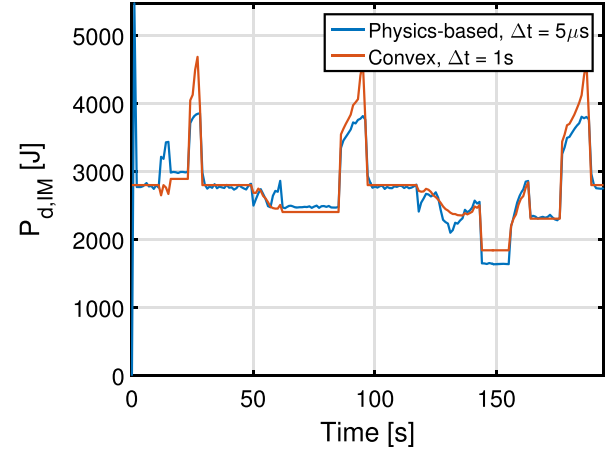

(B) Dissipated power in the electric motor.

FIGURE 10 Evolution of the dissipated power in the battery stack and asynchronous drive over time

\section{5 | CONCLUSION}

Numerical simulation of electrical drivelines proves to be extremely time-consuming, especially in the presence of power electronic elements acting on a very short time scale. Abstracting the individual switching instants and reducing the complexity by focusing on the power transfers within the system allow to reduce the need of computational resources drastically and embed the different time scales in a single representation. The behavior of different electrical components concerning dissipation mechanisms appears to be convex, rendering quadratic, and in general convex, approximations into an appealing modeling alternative. Starting from high-fidelity physics-based models of the various components, capturing all dynamical data concerning the transient behavior, the different loss mechanisms are assessed as these constitute the basis for energy-based calculations and energy management strategies. Both standard losses, ie, dissipation in resistors and parasitic resistances, and power electronic losses, originating from the instantaneous switching events, are taken into consideration. To reduce the complexity of the interconnected system, the interacting information transfer is reduced to power flow representations, for which convex regression models are constructed. For the proposed case study, a reduction in calculation time with a factor 461 is observed, while an accuracy of $98.2 \%$ regarding the assessment of the energy buffer is achieved. The presented convexification procedure copes with the increasing need for computational resources in the simulation of electrical drivetrains and enables complex design problems to become tractable by a proper translation into a convex problem statement. The presented formulation can be directly engaged for energy optimization purposes. The applicability of the proposed methodology is not restricted to vehicle technology solely but can be extended towards a more general notion of mechatronic systems incorporating interacting power transfers.

\section{ACKNOWLEDGEMENT}

The authors acknowledge the support of the Strategic basic research project Emodo of the Flanders Make Strategic Research Centre for the Manufacturing Industry; the BOF01N02716 and BOF 01D37816 project; and the FWO research projectG.0D93.16N. 


\section{ORCID}

\section{Matthias Vandeputte (D) http://orcid.org/0000-0002-6683-3078}

\section{REFERENCES}

1. $\mathrm{CO} 2$ Emissions from fuel combustion highlights. Technical report, International Energy Agency; 2015.

2. Hu X, Murgovski N, Johannesson LM, Egardt B. Comparison of three electrochemical energy buffers applied to a hybrid bus powertrain with simultaneous optimal sizing and energy management. IEEE Trans Intell Transp Syst. 2014;15:1193-1205.

3. Singh B, Singh BN, Chandra A, Al-Haddad K, Pandey A, Kothari DP. A review of single-phase improved power quality AC-DC converters. IEEE Trans Ind Electron. 2003;50:962-981.

4. Bhutia B, Ali SM, Tiadi N. Design of three phase PWM voltage source inverter for photovoltaic application. Int J Innovative Res Electr Electron Instrum Control Eng. 2014;2:1364-1367.

5. Ehsani M, Gao Y, Emadi A. Electric Propulsion Systems. In Modern Electric, Hybrid Electric and Fuel Cell Vehicles: Fundamentels, Theory, and Design. 2nd ed.: Boca Raton: Taylor and Francis Group; 2010.

6. Hiskens IA. Power system modeling for inverse problems. IEEE Transactions on Circuits and Systems. 2004;51(3):539-551.

7. Jimenez JC, Shoji I, Ozaki T. Simulation of stochastic differential equations through the local linearization method. A comparative study. J Stat Phys. 1999;94(3):587-602.

8. Silva E, Hofman T, Steinbuch M. Review of optimal design strategies for hybrid electric vehicles. IFAC Proceedings Volumes. 2012;45(30):57-64.

9. Egardt B, Murgovski N, Pourabdollah M, Mardh LJ. Electromobility studies based on convex optimization design and control issues regarding vehicle electrification. IEEE Control Syst Mag. 2014;34(2):32-49.

10. Shepherd CM. Design of primary and secondary cells: an equation describing battery discharge. J Electrochem Soc. 1965;112:657-664.

11. Tremblay O, Dessaint LA, Dekkiche AI. A generic battery model for the dynamic simulation of hybrid electric vehicles. IEEE Vehicle Power and Propulsion Conference. 2007;1-2:284-289.

12. Salem FA. Controllers and control algorithms: selection and time domain design techniques applied in mechatronics systems design (review and research): part I. International Journal of Engineering Sciences. 2013;2:160-190.

13. Erickson RW, Maksimovic D. Fundamentals of Power Electronics. 2nd ed.: Norwell: Kluwer Academic Publishers; 2004.

14. Irwin JD. Power Electronics Handbook: San Diego: Academic Press; 2001.

15. Nicolai U, Wintrich A. Determining switching losses of Semikron IGBT modules. Technical report, Semikron; 2014.

16. Krause P. Analysis of Electric Machinery and Drive Systems: Institute of Electrical and Electronics Engineers, Hoboken, NJ: John Wiley \& Sons; 2002.

17. Vas P. Sensorless Vector and Direct Torque Control: Oxford: Oxford Science Publications; 1998.

18. Gillespie T. Fundamentals of Vehicle Dynamics: Warrendale: SAE International; 1992.

19. Barlow TJ, Latham S, McCrae IS, Boulter PG. A Reference Book of Driving Cycles for Use in the Measurements of Road Vehicle Emissions: Wokingham: TRL; 2010.

20. Benigni A, Monti A. A parallel approach to real-time simulation of power electronics systems. IEEE Trans Power Electron. 2015;30(9):5192-5206.

21. Tabbache B, Kheloui A, Benbouzid M. Design and control of the induction motor propulsion of an electric vehicle. In: IEEE Vehicle Power and Propulsion Conference, Lille; 2010:1-6.

How to cite this article: Vandeputte M, De Keyser A, Crevecoeur G. Computationally efficient modeling for assessing the energy efficiency of electric drivetrains using convex formulations. Int J Numer Model. 2017;e2275. https://doi.org/10.1002/jnm.2275 